\title{
Membrane-Associated $\alpha$-Tubulin Is Less Acetylated in Postmortem Prefrontal Cortex from Depressed Subjects Relative to Controls: Cytoskeletal Dynamics, HDAC6, and Depression
}

\author{
Harinder Singh, ${ }^{1}$ Justyna Chmura, ${ }^{1}$ Runa Bhaumik, ${ }^{2}$ Ghanshyam N. Pandey, ${ }^{2}$ and ${ }^{\circledR}$ Mark M. Rasenick ${ }^{1,2,3}$ \\ ${ }^{1}$ Department of Physiology and Biophysics, ${ }^{2}$ Department of Psychiatry, University of Illinois at Chicago, Chicago, Illinois 60612, and ${ }^{3}$ Jesse Brown \\ VAMC, Chicago, Illinois 60612
}

Cytoskeletal proteins and post-translational modifications play a role in mood disorders. Post-translational modifications of tubulin also alter microtubule dynamics. Furthermore, tubulin interacts closely with $G \alpha_{s}$, the G-protein responsible for activation of adenylyl cyclase. Postmortem tissue derived from depressed suicide brain showed increased $G \alpha_{s}$ in lipid-raft domains compared with normal subjects. $G \alpha_{s}$, when ensconced in lipid rafts, couples less effectively with adenylyl cyclase to produce cAMP, and this is reversed by antidepressant treatment. A recent in vitro study demonstrated that tubulin anchors $G \alpha_{\mathrm{s}}$ to lipid rafts and that increased tubulin acetylation (due to HDAC6 inhibition) and antidepressant treatment decreased the proportion of $\mathrm{G} \alpha_{s}$ complexed with tubulin. This suggested that deacetylated-tubulin might be more prevalent in depression. This study examined tubulin acetylation in whole-tissue homogenate, plasma membrane, and lipid-raft membrane domains in tissue from normal control subjects, depressed suicides, and depressed nonsuicides (human males/females). While tissue homogenate showed no changes in tubulin acetylation between control, depressed suicides, and depressed nonsuicides, plasma membrane-associated tubulin showed significant decreases in acetylation from depressed suicides and depressed nonsuicides compared with controls. No change was seen in expression of the enzymes responsible for tubulin acetylation or deacetylation. These data suggest that, during depression, membrane-localized tubulin maintains a lower acetylation state, permitting increased sequestration of $G \alpha_{s}$ in lipid-raft domains, where it is less likely to couple to adenylyl cyclase for cAMP production. Thus, membrane tubulin may play a role in mood disorders, which could be exploited for diagnosis and treatment.

Key words: antidepressant; cAMP; cytoskeleton; G-protein; GPCR; lipid-raft

Significance Statement

There is little understanding about the molecular mechanisms involved in the development of depression and, in severe cases, suicide. Evidence for the role of microtubule modifications in progression of depressive disorders is emerging. These postmortem data provide strong evidence for membrane tubulin modification leading to reduced efficacy of the $\mathrm{G}$ protein, $\mathrm{G} \alpha_{\mathrm{s}}$, in depression. This study reveals a direct link between decreased tubulin acetylation in human depression and the increased localization of $\mathrm{G} \alpha_{\mathrm{s}}$ in lipid-raft domains responsible for attenuated cAMP signaling. The evidence presented here suggest a novel diagnostic and therapeutic locus for depression.

\section{Introduction}

Hallmarks of major depressive disorder (MDD) include persistent sad mood, anhedonia, changes in appetite, disturbed sleep, feelings of worthlessness, hopelessness, and suicidal thoughts. While various antidepressant drug therapies are available, the

Received Dec. 23, 2019; revised Feb. 17, 2020; accepted Mar. 12, 2020.

Author contributions: H.S., G.N.P., and M.M.R. designed research; H.S. and J.C. performed research; H.S., R.B., G.N.P., and M.M.R. analyzed data; H.S. wrote the first draft of the paper; H.S., R.B., and M.M.R. wrote the paper; R.B., G.N.P., and M.M.R. edited the paper. biological underpinnings of their action as well as the molecular events leading to depression remain uncertain. Numerous suggestions about the biology of depression exist, and epigenetics (histone deacetylases [HDACs]) and HDAC inhibitors as novel antidepressants are a recent addition to this list (Tsankova et al., 2007; Covington et al., 2009; Gundersen and Blendy, 2009). The

M.M.R. has received research support from Eli Lilly and Lundbeck, and is consultant to Otsuka Pharmaceuticals. He also has ownership in Pax Neuroscience. The remaining authors declare no competing financial interests. 
majority of the presently available antidepressants have, among their actions, prevention of monoamine uptake or degradation, and one consistent effect of antidepressant treatment has been a persistent increase in CAMP and an upregulation of the cAMP generating system (Nibuya et al., 1996; Malberg et al., 2000; Donati and Rasenick, 2003). Furthermore, PET studies from depressed subjects showed global decreases in brain cAMP and antidepressant drugs restored cAMP levels (Fujita et al., 2007, 2017). We have suggested that antidepressants achieve this by a gradual removal of $\mathrm{G} \alpha$ s from lipid rafts and increasing association of that molecule with adenylyl cyclase (Zhang and Rasenick, 2010; Czysz et al., 2015). Consistent with this, postmortem samples from depressed human subjects reveal increased $\mathrm{G} \alpha_{\mathrm{s}}$ (Donati et al., 2008). G $\alpha_{\mathrm{s}}$ is the only heterotrimeric $\mathrm{G}$ protein undergoing translocation out of lipid rafts in response to antidepressant treatment (Toki et al., 1999; Donati and Rasenick, 2005). Interestingly, antidepressant drugs have been shown to concentrate in lipid-raft domains (Eisensamer et al., 2005; Erb et al., 2016). Together, these studies suggest that the lipid environment of $\mathrm{G} \alpha$ s may play an important role in its localization and function, and that chronic antidepressant treatment alters the membrane localization of $\mathrm{G} \alpha$, resulting in augmented coupling to adenylyl cyclase (Allen et al., 2009; Zhang and Rasenick, 2010).

There is evidence for a role of cytoskeletal (microtubules) alterations in the pathology of several neuropsychiatric diseases (Brown et al., 2013; Wong et al., 2013; Scifo et al., 2017). These disorders are associated with structural changes in brain, including synaptic pruning defects and spine and dendrite atrophy (Glausier and Lewis, 2013). The development of depression is associated with exposure to triggering environmental factors, such as chronic stress (Pittenger and Duman, 2008; Lin and Koleske, 2010; Schmitt et al., 2014; McEwen et al., 2015). Most importantly, post-translational modifications, such as acetylation of tubulin, help to maintain cytoskeletal stability (Idriss, 2000; Westermann and Weber, 2003).

Lipid-raft domains are also associated with cytoskeletal elements, such as microtubules. Tubulin is comprised of an $\alpha \beta$ dimer, and these dimers are localized in membranes, and enriched in lipid rafts. Upon activation, $\mathrm{G} \alpha$ s is released form the membrane, where it binds tubulin, activates tubulin GTPase, and increases microtubule dynamics (Roychowdhury and Rasenick, 1994; Davé et al., 2011; Sarma et al., 2015). These findings suggest that tubulin may act as an anchor for $\mathrm{G} \alpha_{\mathrm{s}}$ within the lipidraft domains. A recent in vitro study (Singh et al., 2018) shows that treatment with antidepressants reduces the extent to which $\mathrm{G} \alpha_{\mathrm{s}}$ is complexed with tubulin.

The enzymes responsible for the regulation of acetylation status of $\alpha$-tubulin are HDAC6 (deacetylating) and $\alpha$-tubulin acetyl transferase-1 (ATAT-1: acetylating). There is emerging evidence for the role of HDAC in neuropsychiatric disorders, including MDD (Hobara et al., 2010; Guidotti et al., 2011). Altered levels of HDAC2, 4, 5, 6, and 8 mRNA have been observed in blood cells and postmortem brain from mood disorder subjects (Hubbert et al., 2002; Guidotti et al., 2011). HDAC6, localized in cytosol,

This work was supported by Veterans Affairs Merit Award BX001149 to M.M.R., National Institutes of Health R01AT009169 to M.M.R., National Institutes of Health R21 NS 109862 to M.M.R., National Institutes of Health R01MH106565 to G.N.P., and American Heart Association Postdoctoral Award 16P0ST27770113 to H.S. M.M.R. is a Veterans Affairs Research Career Scientist (BX 004475). We thank Miljiana Petkovic for technical expertise.

Correspondence should be addressed to Mark M. Rasenick at raz@uic.edu.

https://doi.org/10.1523/JNEUROSCI.3033-19.2020

Copyright $\odot 2020$ the authors deacyates $\alpha$-tubulin (Verdel et al., 2000; Hubbert et al., 2002). Peripheral white blood cells derived from MDD subjects showed altered HDAC6 mRNA levels (Hobara et al., 2010).

The current study compares the acetylation status of $\alpha$-tubulin from postmortem human brain of depressed subjects and controls without known psychiatric histories. PFC tissue showed comparable tubulin acetylation in homogenates (Hs), but strikingly decreased acetylation in membranes prepared from depressed suicides (DSs) and depressed nonsuicides (DNSs). These data correspond well with a previous study showing increased $\mathrm{G} \alpha$ s levels in lipid rafts, since acetylation of tubulin decreases its ability to bind $\mathrm{G} \alpha_{\mathrm{s}}$ and anchor it to lipid rafts, resulting in less $\mathrm{G} \alpha_{\mathrm{s}}$ available for adenylyl cyclase activation in the depressed brain. These findings also parallel those of $\mathrm{G} \alpha$ s translocation from lipid rafts by HDAC6 inhibitors (Singh et al., 2018). The data presented here and previous studies in model systems suggest that $\mathrm{G} \alpha$ s anchoring to lipid rafts is involved in both depression and therapies for that disease through modulation of the cAMP-generating system.

These findings suggest a direct role of HDAC6 in maintaining acetylation status of $\alpha$-tubulin, stabilizing/destabilizing microtubules during normal and depressive states. The data also suggest that tubulin acetylation may be relevant to depression and its treatment.

\section{Materials and Methods}

\section{Human subject information}

Tissue used in this study was from Brodmann area 9 obtained from the right hemisphere of DS subjects $(n=15)$, DNS subjects $(n=12)$, and normal control subjects $(n=15)$. Both males and females are included, and subject demographics are described in Table 1. Brain tissues were obtained from the Maryland Brain Collection at the Maryland Psychiatric Research Center. Tissues were collected only after a family member gave informed consent. All procedures were approved by the University of Maryland Institutional Review Board and by the University of Illinois Institutional Review Board.

All tissues from normal controls, DSs, and nonsuicide subjects were screened for evidence of neuropathology. In addition, in each case, screening for the presence of HIV was done in blood samples, and all HIV-positive cases were excluded. Toxicology data were obtained by the analysis of urine and blood samples. $\mathrm{pH}$ of the brain was measured in cerebellum in all cases as described previously (Harrison et al., 1995). Psychiatric drugs in common use as well as drugs of abuse were screened for by using mass spectroscopy. Prescribed drugs were also screened for in interviews.

Control subjects with a known psychiatric illness or a history of alcohol or another drug abuse were excluded. However, alcohol or other substance abuse was present in the MDD subjects as indicated.

\section{Diagnostic method}

Families were queried on all medications or drugs of abuse by trained interviewers. At least one family member, after giving written informed consent, underwent an interview based on the Diagnostic Evaluation After Death, and the Structured Clinical Interview for the DSM-IV (Spitzer et al., 1992). This was done as described in a previous study (Donati et al., 2008).

\section{Sequential detergent extraction of brain membranes}

Brain samples were dissected from the fresh brain and stored at $-80^{\circ} \mathrm{C}$ or dissected from frozen brain tissue with a Stryker autopsy saw, repackaged, and stored at $-80^{\circ} \mathrm{C}$ until use. Brain samples (PFC) were resuspended and minced in TME buffer (10 mm Tris- $\mathrm{HCl}, 1 \mathrm{~mm} \mathrm{MgCl}$, $1 \mathrm{~mm}$ EDTA, pH 7.5, 1 ml/100 mg tissue) followed by homogenization in a motorized Teflon glass homogenizer. Small amount of whole-tissue $\mathrm{H}$ was saved to be run on Western blot along with other cell fractions. The rest of the $\mathrm{H}$ samples were centrifuged at $100,000 \times g$ for $1 \mathrm{~h}$ at $4^{\circ} \mathrm{C}$, and the supernatant (cytosol) and pellet (plasma membrane [PM]) were 
Table 1. Demographic characteristics of suicide and control subjects ${ }^{a}$

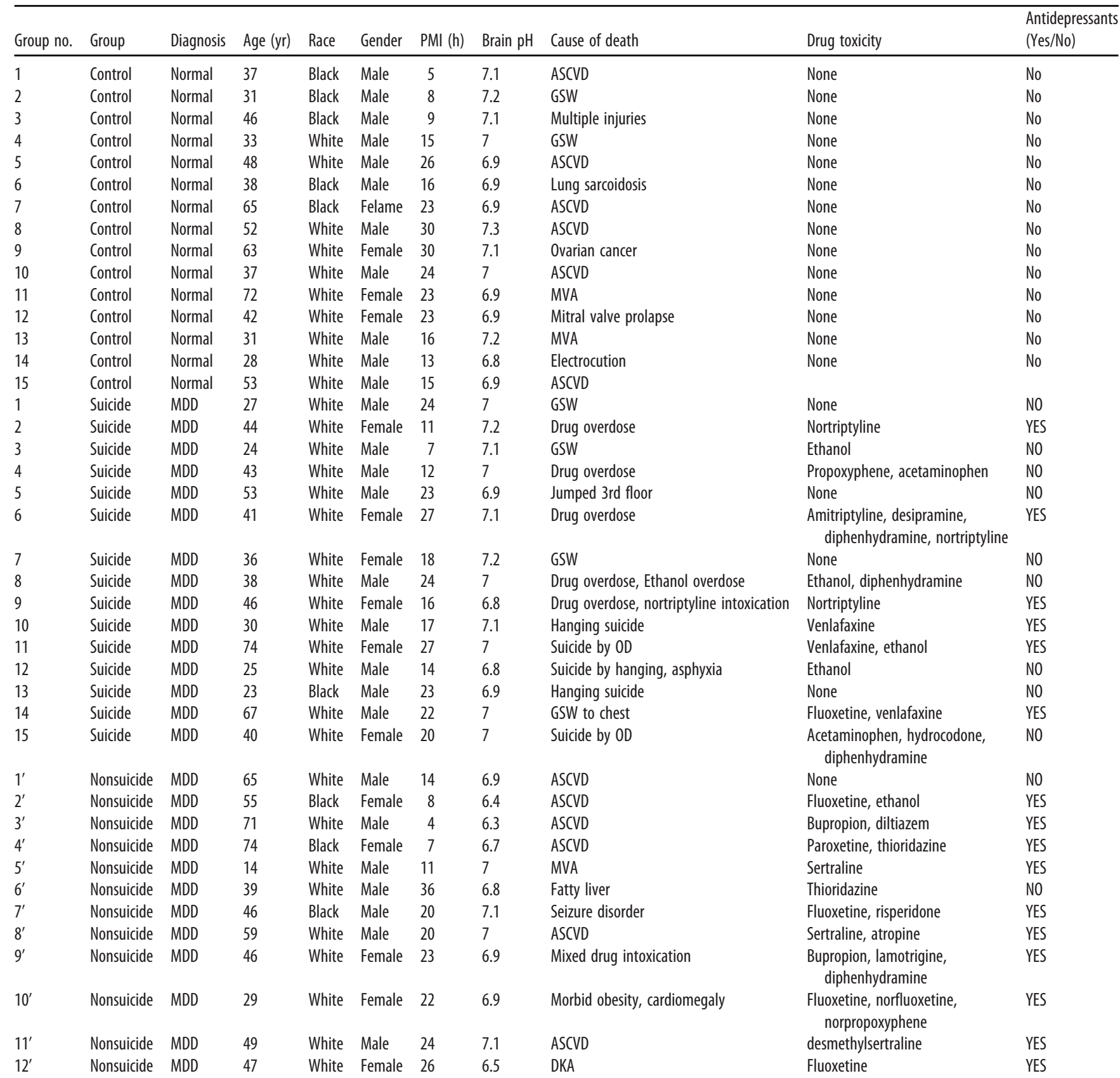

${ }^{a}$ ASCVD, Atherosclerotic cardiovascular disease; GSW, gunshot wound; MVA, motor vehicle accident. Values are Control: \pm SD. Age, $45.07 \pm 13.59$ years; PMI, $18.40 \pm 7.84$ h; brain pH, $7.01 \pm 0.15$; Depressed suicides: Age, $40.73 \pm 15.04$ years; PMI, $19.00 \pm 6.07 \mathrm{~h}$; brain pH, $7.01 \pm 0.12$; Depressed Non-suicides: Age, $58.58 \pm 29.99$ years; PMl, $17.91 \pm 16$ h; brain pH, $6.8 \pm 0.13$.

saved. The crude membrane pellet was extracted with $0.75 \mathrm{ml}$ of TME containing $1 \%$ Triton $\mathrm{X}-100$ for $1 \mathrm{~h}$ at $4^{\circ} \mathrm{C}$ followed by homogenization as above. This sample was centrifuged as above, and both the supernatant (TX-100 extract) and pellet (TX-100-resistant membrane fraction) were saved. This pellet was extracted with $0.75 \mathrm{ml}$ of TME containing $1 \%$ Triton X-114 (TX-114) for $1 \mathrm{~h}$ at $4^{\circ} \mathrm{C}$ and homogenized as above. The sample was centrifuged as above, and both the supernatant (TX-114 extract) and pellet (detergent-insoluble pellet) were saved. The detergent-insoluble pellet could not be efficiently solubilized to be quantified. Herein, the TX-100 extract will be referred to as the TX-100-soluble domain, and the TX-114 extract will be referred to as the TX-100-resistant domain. All fractions were assayed for protein content (Bio-Rad Protein Assay; Bio-Rad) and frozen at $-80^{\circ} \mathrm{C}$ until further use. Frontal cortex was the only brain region available for these experiments (Donati et al., 2008).
SDS-PAGE and Western blotting

Whole-tissue H, PM, TX-100- and TX-114-soluble (TX-100-resistant) membrane fractions $(12-15 \mu \mathrm{g})$ were analyzed by SDS-PAGE followed by Western blotting. The gels were transferred to nitrocellulose membranes (Bio-Rad) by Western blotting. The membranes were blocked with $5 \%$ nonfat dry milk diluted in TBS-T $(10 \mathrm{~mm}$ Tris- $\mathrm{HCl}, 159 \mathrm{~mm}$ $\mathrm{NaCl}$, and $0.1 \%$ Tween $20, \mathrm{pH} 7.4$ ) for $1 \mathrm{~h}$. Following the blocking step, membranes were washed with TBS/Tween 20 and then incubated with an anti-acetyl- $\alpha$-tubulin (Sigma Millipore, \#T7451 clone 6-11B-1), $\alpha$-tubulin (Sigma Millipore, \#T9026), HDAC6 (Cell Signaling Technology, \#7558S), ATAT-1 (Sigma Millipore, \#HPA046816), GAPDH (Proteintech, \#60004-1-Ig) overnight at $4^{\circ} \mathrm{C}$. Membranes were washed with TBS-T and incubated with a secondary antibody [HRP-linked anti-mouse antibody IgG $\mathrm{F}\left(\mathrm{ab}^{\prime}\right) 2$ or HRP-linked anti-rabbit antibody IgG $\mathrm{F}\left(\mathrm{ab}^{\prime}\right) 2$ (Jackson ImmunoResearch Laboratories, catalog \#115-036-072 for mouse, RRID: 
Table 2. ANCOVA results

\begin{tabular}{lllc}
\hline Dependent variable & $\mathrm{df}$ (model, error) & $F$ & $P>F$ \\
\hline Acetyl- $\alpha$-tubulin/total $\alpha$-tubulin (H) & 8,32 & 0.89 & 0.57 \\
Acetyl- $\alpha$-tubulin/total $\alpha$-tubulin (PM) & 8,32 & 2.17 & 0.04 \\
Acetyl- $\alpha$-tubulin/total $\alpha$-tubulin (lipid rafts) & 8,32 & 6.51 & $<0.0001$ \\
\hline
\end{tabular}

AB_2338525) and catalog \#111-036-047 for rabbit, RRID: AB_2337945) for $1 \mathrm{~h}$ at room temperature, washed, and developed using ECL Luminata Forte chemiluminescent reagent (Millipore). Blots were imaged using Chemidoc computerized densitometer (Bio-Rad). The signal intensity of bands from each image were quantitated by densitometry using ImageJ software (National Institutes of Health) and the TX-100-resistant acetyl$\alpha$-tubulin/ $\alpha$-tubulin (TX-114) was compared. The acetyl- $\alpha$-tubulin/ $\alpha$-tubulin were also observed in PM from control (NC), DS, and DNS samples as described (Toki et al., 1999; Donati et al., 2008). Additionally, HDAC6, ATAT-1 and GAPDH expression differences were analyzed between the three groups (C, DS, and DNS).

\section{Normalization}

To be consistent throughout the data collection, the same amount of starting material $(\mathrm{H})$ was used for membrane isolation and lipid-raft extraction. Additionally, GAPDH was used as loading control for all three groups to account for expression differences in $\alpha$-tubulin, HDAC6, and ATAT-1 among groups. Additionally, this normalization procedure was repeated when comparing the amount of acetyl- $\alpha$-tubulin $/ \alpha$-tubulin (normalized densitometry value $=$ sample value $/$ mean value). This allowed us to compare samples accurately among gels and their corresponding blots.

\section{Statistical methods}

Western blot data were analyzed for statistical significance by unpaired, two-tailed Student's $t$ test or one-way ANOVA using Prism 4.0 software package for statistical data analysis (GraphPad). Data are mean \pm SEM, and differences for all experiments were considered significant at $p<0.05\left({ }^{*} p<0.05 ;{ }^{* *} p<0.02\right)$. The differences in TX-114 acetyl$\alpha$-tubulin $/ \alpha$-tubulin, age, gender, $\mathrm{pH}$ of the brain, and postmortem interval (PMI) between depressed and control subjects were analyzed using the independent-sample $t$ test. The relationships between TX-114 acetyl- $\alpha$-tubulin $/ \alpha$-tubulin and PMI, and age were determined by Pearson product-moment correlation analysis. Values of $p$ were twotailed. During data analysis, confounding variables, such as age, PMI, gender, race, antidepressant exposure, and $\mathrm{pH}$ of the brain, were also used as covariates (Proc GLM)(SAS 9.4 statistical software package). A linear model was used to compare NC, DS, and DNS subjects, simultaneously adjusting the effects of age, gender, $\mathrm{PMI}$, brain $\mathrm{pH}$, antidepressant use, ethanol use, nonpsychotropic medicine use, violent suicide, and hypoxia. For post hoc multiple comparisons, we used Bonferroni (Dunn) $t$ tests to adjust the Type I error rates, and we reported mean differences and CI to test the significance at the 0.05 level. In addition, each outcome measure was tested for normality (Kolmogorov-Smirnov) before running the model. All results are included in Tables 2 and 3 .

\section{Results}

There were 11 males and 4 females in the NC group, 9 males and 6 females in the DS group, and 7 males and 5 females in the DNS group (Table 1). The age range was $14-74$ years, whereas the PMI was in the range of 5-30 h. There were no significant differences in age $(t=0.83$; $\mathrm{df}=26 ; p=0.29)$ or PMI $(t=-0.23$; $\mathrm{df}=28 ; p=0.82)$ between suicides and normal control subjects. The mean brain $\mathrm{pH}$ values of NC, DS, and DNS were 7.01 $\pm 0.14,7.01 \pm 0.12$, and $6.8 \pm 0.13$, respectively, which were not different $(t=0.14 ; \mathrm{df}=28$; $p=0.89$ ).

PFC postmortem tissue from control, DS, and DNS subjects showed no changes in acetylation of $\alpha$-tubulin in wholetissue $\mathbf{H}$

The whole-tissue $\mathrm{H}$ sample derived before PM and lipid-raft isolation from PFC tissue of control $(n=15)$, DSs $(n=15)$ and
Table 3. Bonferroni (multiple comparison) results

\begin{tabular}{lllll}
\hline & Group & $\begin{array}{l}\text { Difference } \\
\text { between } \\
\text { DV }\end{array}$ & $\begin{array}{l}\text { Simultaneous } \\
\text { means }\end{array}$ & \multicolumn{1}{l}{$95 \% \mathrm{Cl}$} \\
\hline Acetyl- $\alpha$-tubulin/total & NC vs DS & 0.029 & -0.7510 & 0.8091 \\
$\alpha$-tubulin (H) & NC vs DNS & 0.6143 & -0.2115 & 1.44 \\
& DS vs DNS & 0.5853 & -0.2277 & 1.3982 \\
Acetyl- $\alpha$ tubulin/total & NC vs DNS & 0.5592 & 0.1544 & 0.9640 \\
$\alpha$-tubulin (PM) & NC vs DS & 0.5947 & 0.2124 & 0.9771 \\
& DNS vs DS & 0.0355 & -0.3630 & 0.4340 \\
Acetyl- $\alpha$-tubulin/total & NC vs DS & 3.9386 & 2.4972 & 5.3800 \\
$\alpha$-tubulin (lipid & NC vs DNS & 4.0179 & 2.4920 & 5.5439 \\
rafts) & DS vs DNS & 0.0793 & -1.5816 & 1.4230 \\
\hline
\end{tabular}

DNSs $(n=12)$ showed no changes in acetylated- $\alpha$-tubulin (Fig. $1 A-D)$. The quantification of the results from all three groups (NC, DS, and DNS) showed no significant differences in the extent of tubulin acetylation or any significant effects of covariates, age, gender, $\mathrm{PMI}$, brain $\mathrm{pH}$, antidepressant use, ethanol use, nonpsychotropic medicine use, violent suicide, and hypoxia (Tables 2, 3).

\section{DS brain PM-localized tubulin shows decreased acetylation of $\alpha$-tubulin compared with that of normal controls} PMs isolated from PFC postmortem tissue of NC, DS, and DNS were compared for acetylation status of membrane-associated tubulin. Five samples from each group (NC and DS) were loaded on a single gel (Fig. 2A-C). Additionally, DNS samples (protein concentration equal to NC and DS group subjects) were loaded on a separate gel (Fig. 2D). SDS-PAGE analysis showed significant decrease in acetyl- $\alpha$-tubulin in DS subjects (1-15) and DNS subjects $(n=12)$ compared with the NC subjects. Significant changes were observed between groups in acetyl- $\alpha$-tubulin/ $\alpha$-tubulin $\left(F_{(2)}=8.79, p=0.0009\right)$. The tests from multiple comparisons showed significant differences at 95\% CI between control versus DS (mean difference $=0.59, \mathrm{CI}=(0.21,0.97)$ ) and $\mathrm{NC}$ versus DNS (mean difference $=0.56, \mathrm{CI}=(0.15,0.96)$ ) $($ Fig. $2 E$; Tables 2, 3). There were no significant effects of age, gender, PMI, brain $\mathrm{pH}$, antidepressant use, ethanol use, nonpsychotropic medicine use, violent suicide and hypoxia.

\section{Detergent-resistant/lipid-raft membrane domains as well as TX-114-resistant/nonraft domains show decreased acetylation of $\alpha$-tubulin in depressed subjects compared with normal control postmortem PFC}

Using PM as the starting material (Fig. 2), we isolated lipid-raft fractions to determine whether the decrease in acetylated tubulin was localized to lipid rafts (Fig. 3A-E). The raft domains showed differences in levels of tubulin acetylation. The quantification of the results from all three groups (Control, DS, and DNS) showed significant differences between acetyl- $\alpha$-tubulin $/ \alpha$-tubulin levels in detergent-resistant lipid rafts $\left(F_{(2)}=6.51, p<0.0001\right.$ ) (Fig. $3 F)$. The multiple comparisons between control versus DS subjects and control versus DNSs showed significant differences between the extent of acetyl- $\alpha$-tubulin $/ \alpha$-tubulin in detergent-resistant lipid rafts [Control vs DS (mean difference $=3.94, \mathrm{CI}=$ $(2.49,5.38)$ ), Control vs DNS (mean difference $=4.02, \mathrm{CI}=(2.49$, 5.54))] as shown in Tables 2 and 3. There was a significant effect of hypoxia on lipid-raft tubulin $(t=-2.95, p=0.01)$. There was no effect of age, gender, PMI, brain $\mathrm{pH}$, antidepressant use, ethanol use, nonpsychotropic medicine use, or violent suicide. 
Normal Subjects

A

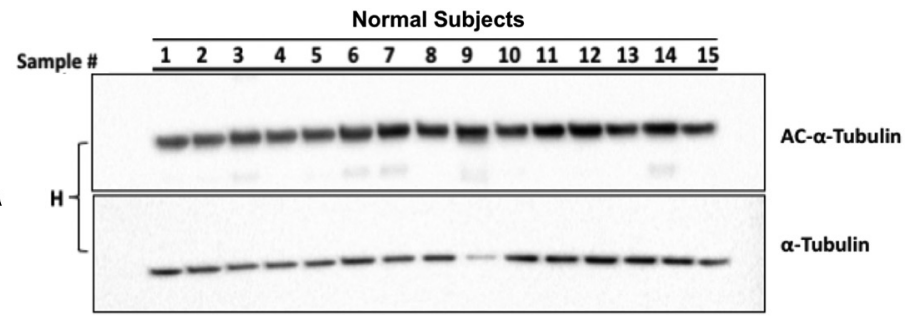

Depressed Suicide Subjects

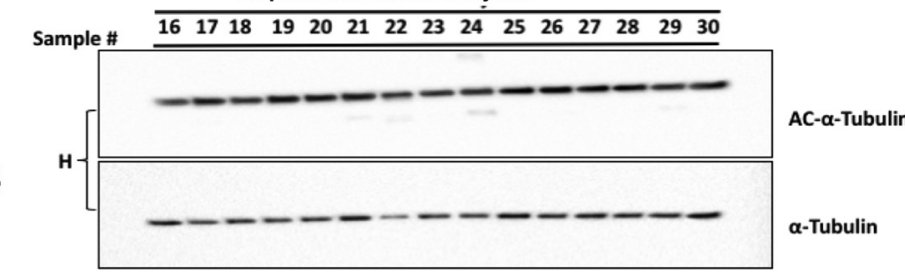

D

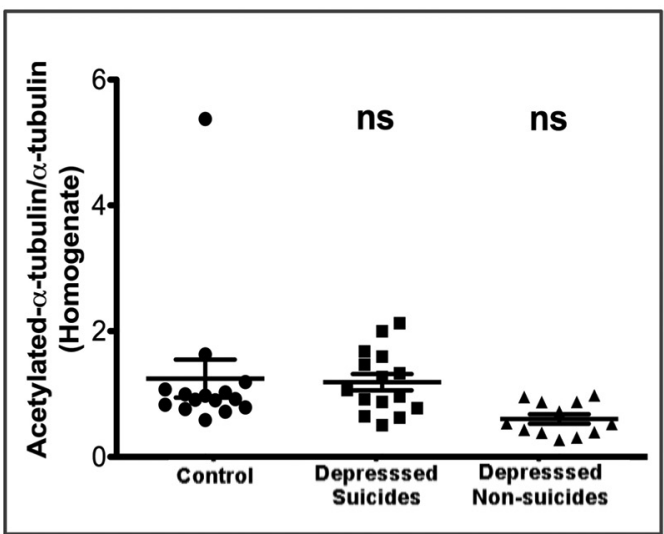

Depressed Non-suicide Subjects

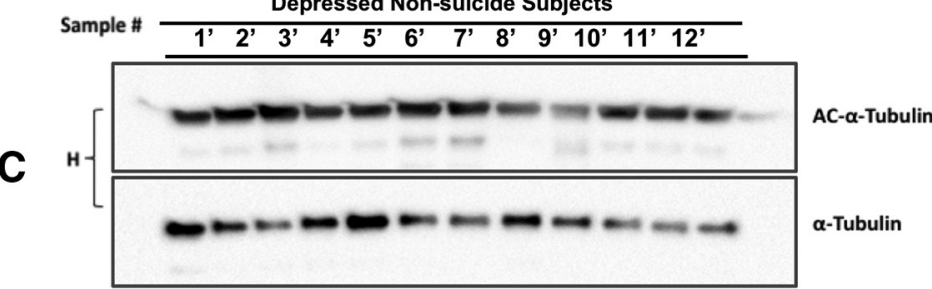

Figure 1. $\alpha$-Tubulin acetylation status in postmortem brain PFC derived from normal and depressed subjects. PFC tissue from $\boldsymbol{A}$ ) control (normal subjects), $\boldsymbol{B}$ ) depressed suicide subjects, and C) Depressed non-suicide subjects was homogenized (H), run on SDSPAGE gels, and transferred to nitrocellulose for detection with either acetyl- $\alpha$-tubulin or $\alpha$-tubulin antibodies. $\boldsymbol{D}$ ) The signal intensity was quantified, and scatter plots were used to show the extent of tubulin acetylation in each group. ns, Nonsignificant compared with control.

A

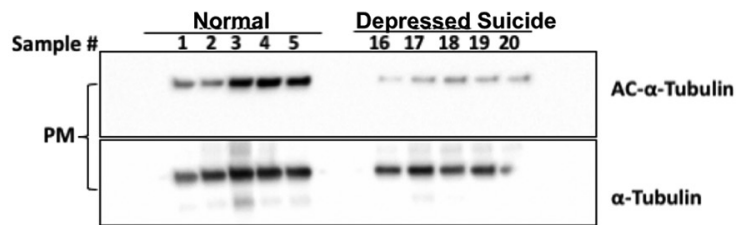

B

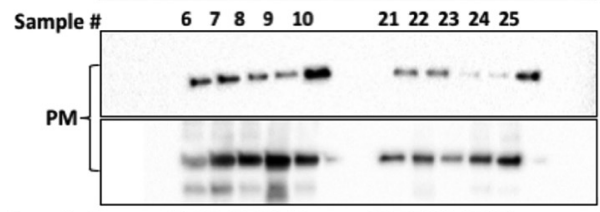

AC- $\alpha$-Tubulin

C

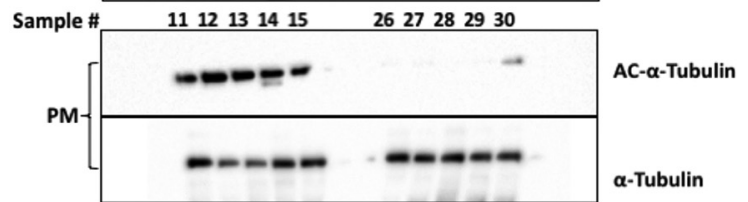

Depressed Non-suicide Subjects

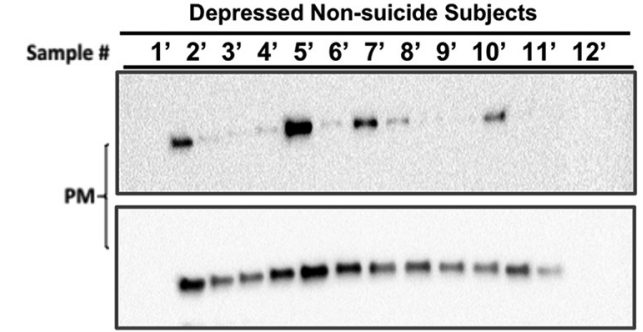

$\mathbf{E}$

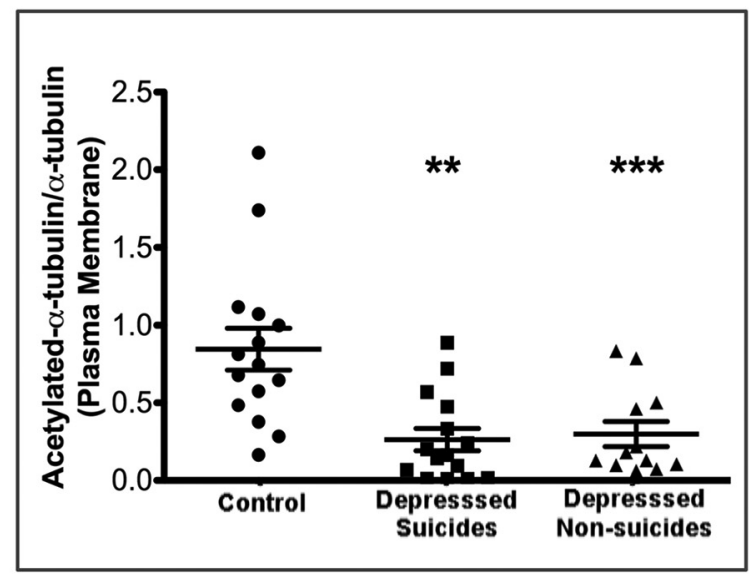

Figure 2. Acetylated tubulin in PM prepared from PFC is decreased in suicides relative to control. PM was isolated from the samples presented in Figure 1 and analyzed in the same manner. PFC tissue from A) control (normal subjects) 1-5 and depressed suicide subjects 16-20, B) subjects 6-10 and 21-25, and C) Subjects 11-15 and 26-30 or D) Depressed non-suicide subjects $1^{\prime}-12^{\prime \prime}$ were analyzed as in Figure 1. E) Scatter plots are used to show the extent of $\alpha$ - tubulin acetylation in both groups. ${ }^{* *} p=0.001 ;{ }^{* * *} p=0.0001$. 

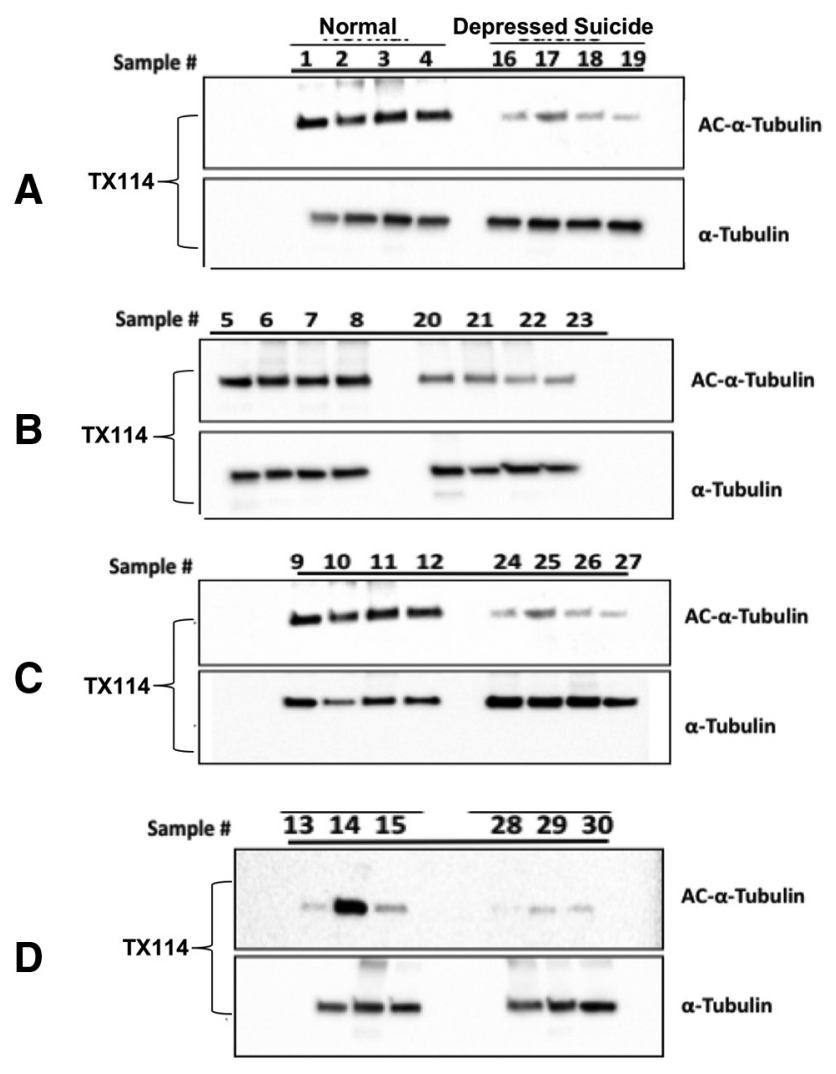

$E$

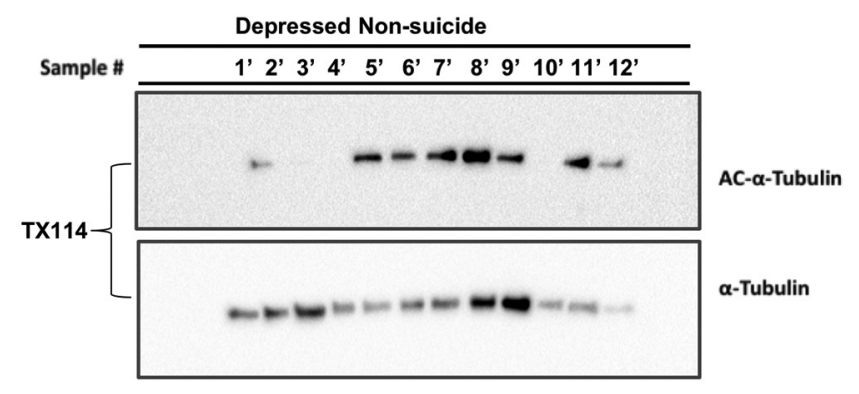

$\mathbf{F}$

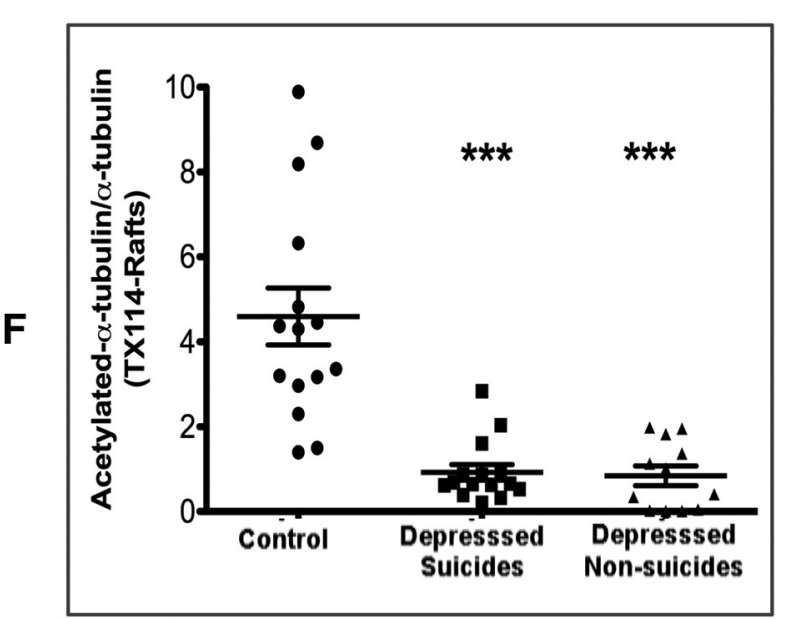

Figure 3. Acetylated tubulin in lipid rafts prepared from postmortem PFC is decreased in depressed subjects relative to control. Plasma membrane was purified, and lipid rafts were prepared by TX-100-resistant (lipid rafts) and TritonX-114-resistant (nonrafts) micro-domain isolation. Samples were analyzed as in Figures 1 and 2. A-D) Shows data for samples isolated from normal controls and depressed suicides. $\boldsymbol{E}$ ) shows samples isolated from depressed non-suicides. $\boldsymbol{F}$ ) Tubulin and acetylated tubulin were quantified as in Figures 1 and 2 . Scatter plots were used to show the extent of tubulin modification. ${ }^{* *} p<0.0001$

\section{Neither tubulin acetylating nor tubulin-deacetylating enzymes show altered expression in depressed brain}

HDAC6 regulates deacetylation of $\alpha$-tubulin, and previous studies in blood cells and postmortem brain tissue derived from patients with mood disorders showed altered HDAC6 expression (Covington et al., 2009). We did not observe these changes (Fig. $4 A-D)$. The enzyme ATAT- 1 specifically acetylates $\alpha$-tubulin at K40, whereas HDAC6 deacetylates. Therefore, along with studying changes in HDAC6 expression levels, we investigated ATAT-1 enzyme level changes. ATAT-1 expression levels/GAPDH remain statistically nonsignificant among NC, DNS, and DS $\left(F_{(2)}=0.96\right.$, $p=0.39$ (Fig. $4 A-C, E$ ). We investigated further the effect of GAPDH or any other covariates on HDAC6 and found no significant effect (Fig. 4D). Similarly, we investigated whether GAPDH and other covariates have any effect on ATAT-1. For one unit increase in GAPDH, ATAT- 1 is increasing by 0.20 unit, but not significantly $(t=0.47, p=0.64)$. Hypoxia $(t=-2.25, p=0.03)$ and violent suicide $(t=2.44, t=0.02)$ have a significant effect on ATAT1. However, there are no group differences in the overall model $\left(F_{(2)}=1.87\right.$, $p=0.17)$. Most importantly, the ATAT1/HDAC6 ratio is not significantly different among the three groups (Fig. $4 F$ ), suggesting that there is no meaningful change in the expression of the enzymes regulating tubulin acetylation.

\section{Discussion}

Postmortem results presented here dovetail well with results in a cellular model revealing that increased tubulin acetylation causes the antidepressant signature response of $\mathrm{G} \alpha$ s translocation from lipid rafts (Singh et al., 2018) Current findings in postmortem brain tissue suggest that acetylation status of tubulin may be important for sequestration of $\mathrm{G} \alpha$ in lipid rafts, as seen in depression (Donati et al., 2008). The findings lend a molecular rationale to antidepressant effects observed in HDAC6-depleted (Espallergues et al., 2012; Fukada et al., 2012; Lee et al., 2012) or pharmacological inhibitor-treated animals (Jochems et al., 2014), where increased tubulin acetylation induced behavioral effects similar to that of traditional antidepressants. While any study relying on immunodetection is subject to variability, the ability to use the comparator of acetylated to total $\alpha$-tubulin lends stability to the data.

The tubulin post-translational modifications observed in postmortem brain tissue from MDD subjects evoke abnormal cytoskeletal organization and disruption of microtubule dynamics, resulting in disrupted neurite growth, synaptogenesis, and dendritic arborization (Wong et al., 2013). Furthermore, proteomic studies from postmortem brain tissue of MDD subjects showed changes in proteins involved in cytoskeletal arrangement, neurotransmission, and synaptic function (Scifo et al., 2017). Chronic stress results in dendritic retraction and synaptic density loss causing regional atrophy in the hippocampus, amygdala, and PFC, as detected in MRI scans of psychiatric patients (McEwen et al., 2015). Finally, there is literature suggesting that microtubules might play a role in mood, memory, and consciousness (Cocchi et al., 2010; Craddock et al., 2012). Based on 

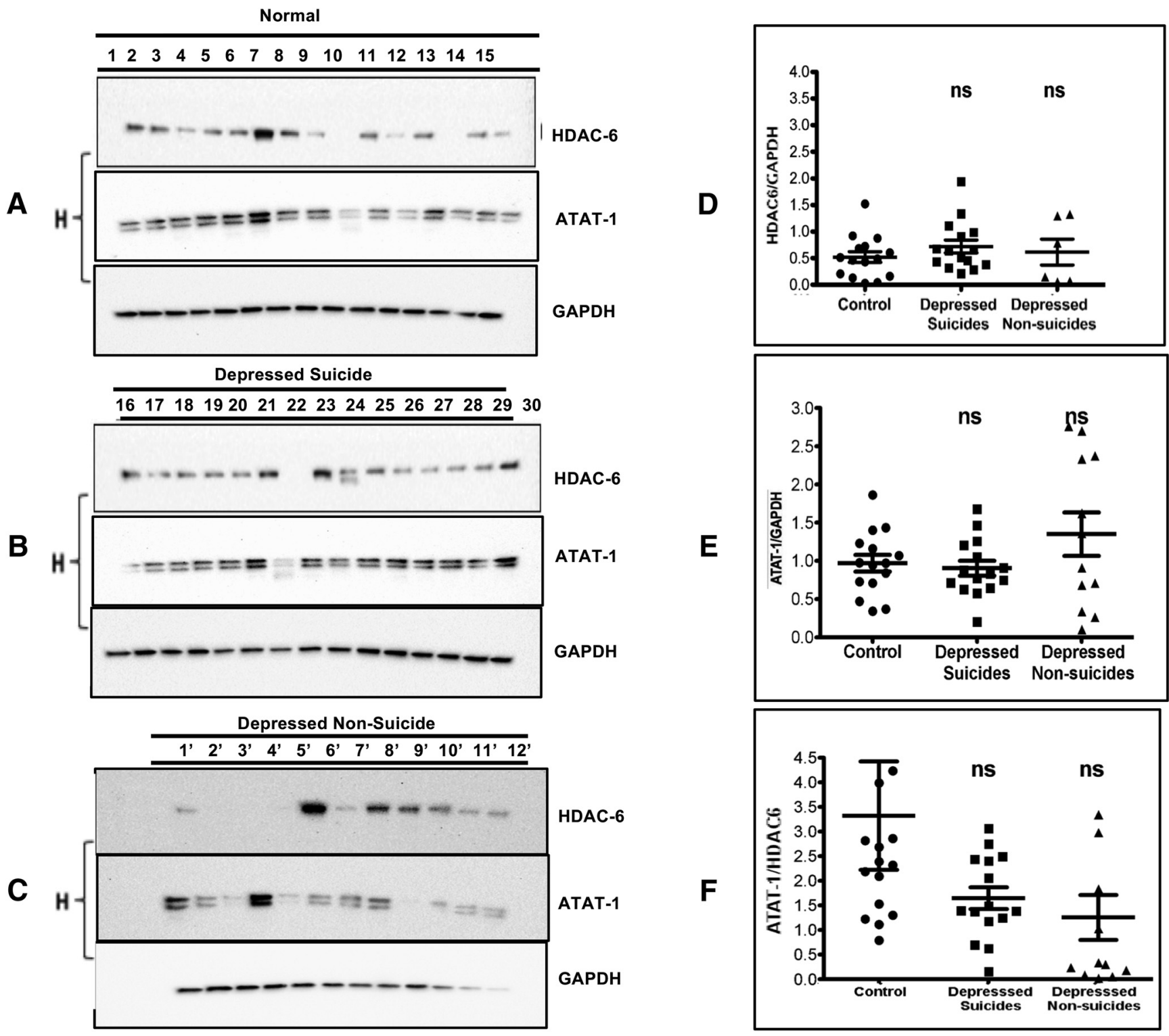

Figure 4. Expression of tubulin acetylating and deacetylating enzymes in postmortem tissue. PF( tissue homogenates from $\boldsymbol{A}$ ) control subjects, $\boldsymbol{B}$ ) depressed suicide subjects and $\boldsymbol{C}$ ) depressed non-suicide subjects were analyzed for presence of ATAT-1 (tubuin-acetylating) and HDAC6 (acetyl tubuiln deacetylating) enzymes using GAPDH as a protein standard. Quantification of $\boldsymbol{D})$ HDAC6/total protein, $\boldsymbol{E}$ ) ATAT1/total protein and $\boldsymbol{F}$ ) HDAC6/ATAT1 is shown. ns, non significant.

these data, altered tubulin and microtubules appear to be a common parameter for several neuropsychiatric disorders.

$\alpha$-Tubulin undergoes acetylation and deacetylation at Lysine40 (K40), catalyzed by acetyl transferase and deacetylase enzymes, respectively. HDAC6, a cytosolic HDAC, is known to deacetylate $\alpha$-tubulin. HDAC6 enzyme is highly expressed in brain, where it is known to regulate emotional behaviors in rodents. HDAC6-deficient mice display hyperactivity, low anxiety, and low depressivelike phenotype, indicating that acetylation status maintains the cellular activity associated with control of emotions (Fukada et al., 2012). Similarly, pharmacological inhibition of HDAC6 in rodents using inhibitors with increased brain bioavailability (ACY738, ACY-775) shows increased anxiolytic and antidepressant-like effects in mice undergoing "depression-inducing" paradigms (Jochems et al., 2014). Furthermore, chronic stress in rodents has been shown to induce increased expression of HDAC6 in hippocampus (Jianhua et al., 2017). Decreased levels of acetylated tubulin are found in the hippocampus of rats following social isolation (Bianchi et al., 2009). These studies further corroborated the microtubule roles, especially tubulin acetylation, in the pathophysiology of depression. Decreased dendritic spine density and reduced dendritic arborization are associated with neurologic diseases (Blanpied and Ehlers, 2004; Penzes and Vanleeuwen, 2011), including intellectual disability (Kaufmann et al., 2000), depression (Duman and Canli, 2015), and schizophrenia (Penzes and Vanleeuwen, 2011; Glausier and Lewis, 2013). Chronic stress induces atrophy in hippocampus and PFC, areas important for mood regulation. Reduced dendritic field size results in abrogated synaptogenesis (Gold, 2015). HDAC6 regulates deacetylation of $\alpha$-tubulin, and previous studies in blood cells and postmortem brain tissue derived from patients with mood disorders showed altered HDAC6 expression (Covington et al., 2009). Post-translational modifications in $\alpha$-tubulin (acetyl- $\alpha$-tubulin) result from either increased enzyme expression or increased enzyme activity. We did not observe any specific expression pattern within each group or among three groups when normalized 
to total $\alpha$-tubulin (Control, DS, DNS). The enzyme ATAT- 1 specifically acetylates the $\alpha$-tubulin at K-40, acting as the "accelerator" to the "brake" represented by HDAC6. ATAT-1 expression levels show no significant difference among control, DSs and DNSs $\left(F_{(8,32)}=1.04, p=0.43\right)$ (Fig. 4). Nonetheless, results in Figures 2 and 3 reveal that depressed subjects show decreased acetylated a tubulin in membrane fractions. This suggests that the activity of HDAC6 relative to ATAT1 is increased without any change in the expression of either enzyme. This could be explained by multiple factors. First, HDAC6 is regulated by nitrosylation (Okuda et al., 2015). Perhaps more importantly, only membrane tubulin (particularly lipid-raft tubulin) was affected, as the total degree of tubulin acetylation was constant among all groups. Perhaps some membrane translocating mechanism is at play.

These findings are consistent with a link between decreased $\alpha$-tubulin acetylation and increased localization of $\mathrm{G} \alpha$ s in lipid rafts. Our in vitro studies in C6 cells showing HDAC6 inhibition induced $\alpha$-tubulin acetylation results in disruption of tubulin$\mathrm{G} \alpha_{\mathrm{s}}$ complex, specifically in the lipid-raft domain, bolster this (Singh et al., 2018). Furthermore, membrane tubulin appears to be associated, preferentially, with lipid rafts (Ishmael et al., 2007), so membrane tubulin and lipid-raft tubulin may be identical. While earlier studies showed that tubulin binding to $\mathrm{G} \alpha$ s was sensitive to $\mathrm{G} \alpha_{\mathrm{s}}$ conformation, the nucleotide status of tubulin was not important (Yu et al., 1999). The apparent binding site for $\mathrm{G} \alpha_{\mathrm{s}}$ on tubulin involves the $\alpha 3 \beta 5$ region of $\mathrm{G} \alpha_{\mathrm{s}}$ and the GTP-binding pocket of $\alpha$-tubulin (Layden et al., 2008; Davé et al., 2011) While the structural changes to $\alpha$-tubulin resulting from modifying $\alpha$-tubulin have not been established, it is clear that modifying $\alpha$-tubulin has structural implications for the dimer (Nogales et al., 1998).

This study also is consistent with depression reducing availability of $\mathrm{G} \alpha$ s to activate adenylyl cyclase and a resultant decrease in cAMP production (Donati et al., 2008; Fujita et al., 2017). While those studies represent postmortem and PET imaging in human brain, human peripheral tissue from depressed subjects (platelets and lymphocytes) also shows diminished $\mathrm{G} \alpha_{\mathrm{s} \text { s }}$ stimulated adenylyl cyclase in depression (Pandey et al., 1985; Hines and Tabakoff, 2005; Mooney et al., 2013). Three of these studies above examined subjects before and after antidepressant treatment, and in those subjects responding to treatment, $\mathrm{G} \alpha_{\mathrm{s}}$-stimulated cAMP production returned to levels seen in healthy controls (Pandey et al., 1985; Fujita et al., 2017; Mooney et al., 2013). Mice susceptible to stress show decreased cAMP and greater raft localization of $\mathrm{G} \alpha_{\mathrm{s}}$ in their nucleus accumbens and increasing cAMP in that brain region has an "antidepressant" effect (Zhang et al., 2020). Consistent with this, sustained treatment of cultured neuronal or glial cells with antidepressants translocates $\mathrm{G} \alpha$ s from lipid rafts and increases $\mathrm{G} \alpha_{\mathrm{s}}$-activated cAMP (Donati and Rasenick, 2005; Czysz et al., 2015; Singh et al., 2018). Ketamine also has this effect, but on an accelerated time scale (Wray et al., 2019).

Several of the subjects on this study showed evidence of antidepressants in their blood. Some subjects were prescribed these drugs and others may have ingested them, along with other drugs, in the course of their suicide. Regardless, there was no effect of antidepressants on tubulin acetylation (or $\mathrm{G} \alpha_{\mathrm{s}}$ in lipid rafts) (Donati et al., 2008). Given the observation that, absent therapeutic effect, antidepressants did not increase cAMP, the lack of effect on tubulin acetylation or raft association of $\mathrm{G} \alpha_{\mathrm{s}}$ is consistent. Certainly antidepressant treatment translocates $\mathrm{G} \alpha$ s from lipid rafts in cultured cells or rodents, While neither cells nor rodents were, necessarily, "depressed," antidepressants commonly show a legion of behavioral, cellular, and neurophysiological effects.

This study strikes a thematic note in revealing that compounds with antidepressant activity show a consistent "biosignature" in the release of $\mathrm{G} \alpha_{\mathrm{s}}$ from lipid rafts and the subsequent association of that molecule with adenylyl cyclase, evoking a sustained increase in cellular cAMP (Singh et al., 2018). We have also demonstrated that increased acetylation of tubulin can explain this, in part. Furthermore, the diminished tubulin acetylation seen in lipid rafts from depressed subjects might explain the increase in $\mathrm{G} \alpha_{\mathrm{s}}$ seen in their lipid rafts. Nevertheless, the ability of monoamine-centered antidepressants to mitigate $\mathrm{G} \alpha_{\mathrm{s}}$-tubulin association without altering tubulin acetylation (Singh et al., 2018) argues for the complexity of depression and its therapy.

\section{References}

Allen JA, Yu JZ, Davé RH, Bhatnagar A, Roth BL, Rasenick MM (2009) Caveolin-1 and lipid microdomains regulate Gs trafficking and attenuate Gs/adenylyl cyclase signaling. Mol Pharmacol 76:1082-1093.

Bianchi M, Shah AJ, Fone KC, Atkins AR, Dawson LA, Heidbreder CA, Hows ME, Hagan JJ, Marsden CA (2009) Fluoxetine administration modulates the cytoskeletal microtubular system in the rat hippocampus. Synapse 63:359-364.

Blanpied TA, Ehlers MD (2004) Microanatomy of dendritic spines: emerging principles of synaptic pathology in psychiatric and neurological disease. Biol Psychiatry 55:1121-1127.

Brown AS, Borgmann-Winter K, Hahn CG, Role L, Talmage D, Gur R, Chow J, Prado P, McCloskey T, Bao Y, Bulinski JC, Dwork AJ (2013) Increased stability of microtubules in cultured olfactory neuroepithelial cells from individuals with schizophrenia. Prog Neuropsychopharmacol Biol Psychiatry 48:1252-258.

Cocchi M, Tonello L, Rasenick MM (2010) Human depression: a new approach in quantitative psychiatry. Ann Gen Psychiatry 9:25.

Covington HE, Maze I, LaPlant QC, Vialou VF, Ohnishi YN, Berton O, Fass DM, Renthal W, Rush AJ, Wu EY, Ghose S, Krishnan V, Russo SJ, Tamminga C, Haggarty SJ, Nestler EJ (2009) Antidepressant actions of histone deacetylase inhibitors. J Neurosci 29:11451-11460.

Craddock T, Tuszynski J, Hameroff S (2012) Cytoskeletal signaling: is memory encoded in microtubule lattices. PLoS Comput Biol 8:e1002421.

Czysz AH, Schappi JM, Rasenick MM (2015) Lateral diffusion of G $\alpha$ s in the plasma membrane is decreased after chronic but not acute antidepressant treatment: role of lipid raft and non-raft membrane microdomains. Neuropsychopharmacology 40:766-773.

Davé RH, Saengsawang W, Lopus M, Davé S, Wilson L, Rasenick MM (2011) A molecular and structural mechanism for G protein-mediated microtubule destabilization. J Biol Chem 286:4319-4328.

Donati RJ, Rasenick MM (2003) G protein signaling and the molecular basis of antidepressant action. Life Sci 73:1-17.

Donati RJ, Rasenick MM (2005) Chronic antidepressant treatment prevents accumulation of Gsalpha in cholesterol-rich, cytoskeletal-associated, plasma membrane domains (lipid rafts). Neuropsychopharmacology 30:1238-1245.

Donati RJ, Dwivedi Y, Roberts RC, Conley RR, Pandey GN, Rasenick MM (2008) Postmortem brain tissue of depressed suicides reveals increased Gs alpha localization in lipid-raft domains where it is less likely to activate adenylyl cyclase. J Neurosci 28:3042-3050.

Duman EA, Canli T (2015) Influence of life stress, 5-HTTLPR genotype, and SLC6A4 methylation on gene expression and stress response in healthy Caucasian males. Biol Mood Anxiety Disord 5:2.

Eisensamer B, Uhr M, Meyr S, Gimpl G, Deiml T, Rammes G, Lambert JJ, Zieglgansberger W, Holsboer F, Rupprecht R (2005) Antidepressants and antipsychotic drugs colocalize with 5-HT3 receptors in raft-like domains. J Neurosci 25:10198-10206.

Erb SJ, Schappi JM, Rasenick MM (2016) Antidepressants accumulate in lipid rafts independent of monoamine transporters to modulate redistribution of the G protein, Galphas. J Biol Chem 291:19725-19733.

Espallergues J, Teegarden SL, Veerakumar A, Boulden J, Challis C, Jochems J, Chan M, Petersen T, Deneris E, Matthias P, Hahn CG, Lucki I, Beck 
SG, Berton O (2012) HDAC6 regulates glucocorticoid receptor signaling in serotonin pathways with critical impact on stress resilience. J Neurosci 32:4400-4416

Fujita M, Richards EM, Niciu MJ, Ionescu DF, Zoghbi SS, Hong J, Telu S, Hines CS, Pike VW, Zarate CA, Innis RB (2017) cAMP signaling in brain is decreased in unmedicated depressed patients and increased by treatment with a selective serotonin reuptake inhibitor. Mol Psychiatry 22:754-759.

Fujita M, Imaizumi M, D’Sa C, Zoghbi SS, Crescenzo MS, Hong J, Musachio JL, Gee AD, Seidel J, Green MV, Pike VW, Duman RS, Innis RB (2007) In vivo and in vitro measurement of brain phosphodiesterase 4 in rats after antidepressant administration. Synapse 61:78-86.

Fukada M, Hanai A, Nakayama A, Suzuki T, Miyata N, Rodriguiz RM, Wetsel WC, Yao TP, Kawaguchi Y (2012) Loss of deacetylation activity of Hdac6 affects emotional behavior in mice. PLoS One 7:e30924.

Glausier JR, Lewis DA (2013) Dendritic spine pathology in schizophrenia. Neuroscience 251:90-107.

Gold PW (2015) The organization of the stress system and its dysregulation in depressive illness. Mol Psychiatry 20:32-47.

Guidotti A, Auta J, Chen Y, Davis JM, Dong E, Gavin DP, Grayson DR, Matrisciano F, Pinna G, Satta R, Sharma RP, Tremolizzo L, Tueting P (2011) Epigenetic GABAergic targets in schizophrenia and bipolar disorder. Neuropharmacology 60:1007-1116.

Gundersen BB, Blendy JA (2009) Effects of the histone deacetylase inhibitor sodium butyrate in models of depression and anxiety. Neuropharmacology 57:67-74.

Harrison PJ, Heath PR, Eastwood SL, Burnet PW, McDonald B, Pearson RC (1995) The relative importance of premortem acidosis and postmortem interval for human brain gene expression studies: selective mRNA vulnerability and comparison with their encoded proteins. Neurosci Lett 200:151-154.

Hines LM, Tabakoff B (2005) Platelet adenylyl cyclase activity: a biological marker for major depression and recent drug use. Biol Psychiatry 58:955-962.

Hobara T, Uchida S, Otsuki K, Matsubara T, Funato H, Matsuo K, Suetsugi M, Watanabe Y (2010) Altered gene expression of histone deacetylases in mood disorder patients. J Psychiatr Res 44:263-270.

Hubbert C, Guardiola A, Shao R, Kawaguchi Y, Ito A, Nixon A, Yoshida M, Wang XF, Yao TP (2002) HDAC6 is a microtubule-associated deacetylase. Nature 417:455-458.

Idriss HT (2000) Phosphorylation of tubulin tyrosine ligase: a potential mechanism for regulation of alpha-tubulin tyrosination. Cell Motil Cytoskeleton 46:1-5.

Ishmael JE, Safic M, Amparan D, Vogel WK, Pham T, Marley K, Filtz TM, Maier CS (2007) Nonmuscle myosins II-B and Va are components of detergent-resistant membrane skeletons derived from mouse forebrain. Brain Res 1143:46-59.

Jianhua F, Wei W, Xiaomei L, Shao-Hui W (2017) Chronic social defeat stress leads to changes of behaviour and memory-associated proteins of young mice. Behav Brain Res 316:136-144.

Jochems J, Boulden J, Lee BG, Blendy JA, Jarpe M, Mazitschek R, Van Duzer JH, Jones S, Berton O (2014) Antidepressant-like properties of novel HDAC6-selective inhibitors with improved brain bioavailability. Neuropsychopharmacology 39:389-400.

Kaufmann WE, MacDonald SM, Altamura CR (2000) Dendritic cytoskeletal protein expression in mental retardation: an immunohistochemical study of the neocortex in Rett syndrome. Cereb Cortex 10:992-1004.

Layden BT, Saengsawang W, Donati RJ, Yang S, Mulhearn DC, Johnson ME, Rasenick MM (2008) Structural model of a complex between the heterotrimeric G protein, Gsalpha, and tubulin. Biochim Biophys Acta 1783:964-973.

Lee JB, Wei J, Liu W, Cheng J, Feng J, Yan Z (2012) Histone deacetylase 6 gates the synaptic action of acute stress in prefrontal cortex. J Physiol 590:1535-1546

Lin YC, Koleske AJ (2010) Mechanisms of synapse and dendrite maintenance and their disruption in psychiatric and neurodegenerative disorders. Annu Rev Neurosci 33:349-378.

Okuda K, Ito A, Uehara T (2015) Regulation of Histone Deacetylase 6 Activity via S-Nitrosylation. Biol Pharm Bull 38:1434-1437.
Malberg JE, Eisch AJ, Nestler EJ, Duman RS (2000) Chronic antidepressant treatment increases neurogenesis in adult rat hippocampus. J Neurosci 20:9104-9110.

McEwen BS, Bowles NP, Gray JD, Hill MN, Hunter RG, Karatsoreos IN, Nasca C (2015) Mechanisms of stress in the brain. Nat Neurosci 18:1353-1363.

Mooney JJ, Samson JA, McHale NL, Pappalarado KM, Alpert JE, Schildkraut JJ (2013) Increased Gsa within blood cells membrane lipid microdomains in some depressive disorders: an exploratory study. J Psychiatr Res 47:706-711.

Nibuya M, Nestler EJ, Duman RS (1996) Chronic antidepressant administration increases the expression of cAMP response element binding protein (CREB) in rat hippocampus. J Neurosci 16:2365-2372.

Nogales E, Wolf SG, Downing KH (1998) Structure of the alpha beta tubulin dimer by electron crystallography. Nature 391:199-203.

Pandey GN, Janicak P, Davis JM (1985) Studies of beta-adrenergic receptors in leukocytes of patients with affective illness and effects of antidepressant drugs. Psychopharmacol Bull 21:603-609.

Penzes P, Vanleeuwen JE (2011) Impaired regulation of synaptic actin cytoskeleton in Alzheimer's disease. Brain Res Rev 67:184-192.

Pittenger C, Duman RS (2008) Stress, depression, and neuroplasticity: a convergence of mechanisms. Neuropsychopharmacology 33:88-109.

Roychowdhury S, Rasenick MM (1994) Tubulin-G protein association stabilizes GTP binding and activates GTPase: cytoskeletal participation in neuronal signal transduction. Biochemistry 33:9800-9805.

Sarma T, Koutsouris A, Yu JZ, Krbanjevic A, Hope TJ, Rasenick MM (2015) Activation of microtubule dynamics increases neuronal growth via the nerve growth factor (NGF)- and Galphas-mediated signaling pathways. J Biol Chem 290:10045-10056.

Schmitt A, Malchow B, Hasan A, Falkai P (2014) The impact of environmental factors in severe psychiatric disorders. Front Neurosci 8:19.

Scifo E, Pabba M, Kapadia F, Ma T, Lewis DA, Tseng GC, Sibille E (2017) Sustained molecular pathology across episodes and remission in major depressive disorder. Biol Psychiatry 83:81-89.

Singh H, Wray N, Schappi JM, Rasenick MM (2018) Disruption of lipid-raft localized Galphas/tubulin complexes by antidepressants: a unique feature of HDAC6 inhibitors, SSRI and tricyclic compounds. Neuropsychopharmacology 43:1481-1491.

Spitzer RL, Williams JB, Gibbon M, First MB (1992) The Structured Clinical Interview for DSM-III-R (SCID): I. History, rationale, and description. Arch Gen Psychiatry 49:624-629.

Toki S, Donati RJ, Rasenick MM (1999) Treatment of C6 glioma cells and rats with antidepressant drugs increases the detergent extraction of $\mathrm{G}(\mathrm{s}$ alpha) from plasma membrane. J Neurochem 73:1114-1120.

Tsankova N, Renthal W, Kumar A, Nestler EJ (2007) Epigenetic regulation in psychiatric disorders. Nat Rev Neurosci 8:355-367.

Verdel A, Curtet S, Brocard MP, Rousseaux S, Lemercier C, Yoshida M, Khochbin S (2000) Active maintenance of mHDA2/mHDAC6 histonedeacetylase in the cytoplasm. Curr Biol 10:747-749.

Westermann S, Weber K (2003) Post-translational modifications regulate microtubule function. Nat Rev Mol Cell Biol 4:938-947.

Wong GT, Chang RC, Law AC (2013) A breach in the scaffold: the possible role of cytoskeleton dysfunction in the pathogenesis of major depression. Ageing Res Rev 12:67-75.

Wray N, Schappi J, Singh H, Senese N, Rasenick MM (2019) NMDA receptor-independent, cAMP- dependent antidepressant actions of ketamine. Mol Psychiatry 24:1833-1843.

Yu XC, Margolin W, Gonzalez-Garay ML, Cabral F (1999) Vinblastine induces an interaction between FtsZ and tubulin in mammalian cells. J Cell Sci 112:2301-2311.

Zhang L, Rasenick MM (2010) Chronic treatment with escitalopram but not R-citalopram translocates Galpha(s) from lipid-raft domains and potentiates adenylyl cyclase: a 5-hydroxytryptamine transporter-independent action of this antidepressant compound. J Pharmacol Exp Ther 332:977984.

Zhang Y, Lu W, Wang Z, Zhang R, Xie Y, Guo S, Jiao L, Hong Y, Di Z, Wang G, Aa J (2020) Reduced neuronal cAMP in the nucleus accumbens damages blood-brain barrier integrity and promotes stress vulnerability. Biol Psychiatry 87:526-537. 